\title{
Modelagem, Simulação e Controle do Sistema de Abastecimento de Água do Campus da Universidade Federal do Rio Grande do Norte
}

\author{
Eduardo Nogueira Cunha ${ }^{1}$ \\ Instituto Metrópole Digital, UFRN, Natal, RN. \\ Carlos Eduardo Trabuco Dórea ${ }^{2}$ \\ Departamento de Engenharia de Computação e Automação, UFRN, Natal, RN.
}

\begin{abstract}
Resumo. O artigo apresenta a modelagem matemática, simulação e controle do sistema de abastecimento de água (SAA) do campus da Universidade Federal do Rio grande do Norte (UFRN). Na simulação é apresentado a resposta do sistema modelado em malha aberta e em malha fechada usando o controlador servossistema do tipo 1 aplicado a uma planta multivariável.

Palavras-chave. Modelagem Matemática, Controle de Processos, Sistema de Abastecimento de Água.
\end{abstract}

\section{Introdução}

Hoje o Brasil é o $20^{\circ}$ país em perda de água tratada no mundo [3], o país perde $39 \%$ de toda a agua disponibilizada para distribuir para a sociedade. No Rio Grande do Norte, segundo [3], esse número é ainda maior, perdemos cerca de $47 \%$ da água disponibilizada para consumo humano. Grande parte dessa perda se dá pela falta de controle, levando a uma má distribuição, transbordamento de reservatórios, vazamentos devido elevada pressão nas tubulações. Os SAA são críticos e não existe possibilidade de teste e paradas para simulações na planta real. O objetivo desse trabalho é obter um modelo matemático para o sistema que possa descrever bem sua dinâmica, para que através de simulações possamos obter e testar uma estratégia de controle para ser aplicada no controlador para um sistema multivariável.

\section{Modelagem do Sistema de Abastecimento de Água da UFRN}

O campus da UFRN é composto por cinco zonas de abastecimento, em cada zona temos sistemas independentes de abastecimento por área, mas interligados por uma rede

1 eduardo@imd.ufrn.br

2 cetdorea@dca.ufrn.br 
auxiliar entre todos os sete reservatórios.

A água é captada na própria zona de abastecimento, através de poços tubulares com vazão de $20 \mathrm{~m}^{3} / \mathrm{h}$, exceto a zona dois onde há dois poços com vazão de $10 \mathrm{~m}^{3} / \mathrm{h}$. A reserva de água é feita nos reservatórios existentes em cada zona, com exceção a zona três que possui três reservatórios. A capacidade de armazenamento dos reservatórios é de $200 \mathrm{~m}^{3}$, com um nível dinâmico de $6 \mathrm{~m}$. Toda distribuição de água no campus é feita através de tubulações de PVC com diâmetros variando de acordo com a área que se quer abastecer. Nas saídas dos reservatórios onde estão instaladas as válvulas de controle, o diâmetro da tubulação é de $100 \mathrm{~mm}$, o que corresponde também ao diâmetro das válvulas que controlam tanto a interligação da malha de abastecimento, como a ligação com a malha que conecta todos os reservatórios como mostrado na figura 1.

\subsection{Componentes do sistema}

Nesse trabalho vamos dar ênfase aos elementos que compõem a dinâmica do sistema que desejamos estudar.

\subsubsection{Bombas}

As bombas são elementos responsáveis por transformar a energia desenvolvida no motor elétrico em energia potencial em seu eixo. A velocidade $n(R P M)$ no eixo da bomba varia não linearmente com a velocidade $N(R P M)$ no eixo do motor e com vazão $Q_{p}(t)\left(\mathrm{m}^{3} / \mathrm{s}\right)$. A altura manométrica desenvolvida pela bomba $h_{B}$ pode ser escrita em função de $N, Q_{p}$ como é visto em [2] da seguinte forma:

$$
h_{B}\left(N, Q_{p}\right)=A_{0} \mathrm{~N}^{2}+\frac{B_{0}}{n} N Q_{p}-\frac{C_{0}}{n^{2}} Q_{p}^{2}
$$

Onde, $A_{0}, B_{0}$ e $C_{0}$ são constantes da bomba, e dependem das suas características de fabricação.

\subsubsection{Reservatórios}

Os reservatórios são largamente utilizados em sistemas de abastecimento de água, tanto para reservar a água quando para manter o equilíbrio de pressão no sistema. Utilizando o princípio da equação da continuidade temos que a variação do volume $V$ é dada por:

$$
\rho \frac{d V(t)}{d t}=\rho_{i} Q_{i}(t)-\rho_{0} Q_{0}(t)
$$

Onde, $\rho, \rho_{i}$ e $\rho_{0}$ são a densidade especifica da água no reservatório. Como toda água que entra no reservatório tende a sair podemos dizer que $\rho=\rho_{i}=\rho_{0}$.

Como base em relações geométricas temos que o volume do reservatório $V\left(\mathrm{~m}^{3}\right)$ pode ser dado também em função da área da sua base $A_{b}(m)$ e da altura (nível $h_{t}(m)$ ), assim:

$$
V(t)=A_{b} h_{t}(t)
$$

Substituindo (3) em (2) temos, 


$$
\frac{d h_{t}(t)}{d t}=\frac{Q_{i}(t)-Q_{0}(t)}{A_{b}}
$$

\subsubsection{Velocidade de escoamento}

O teorema de Bernoulli descreve a relação da velocidade $v(\mathrm{~m} / \mathrm{s})$ de um fluido em uma tubulação saindo de um reservatório e o nível do reservatório $h(m)$, como:

$$
v(t)=\sqrt{2 g h(t)}
$$

Onde, $g$ é a aceleração da gravidade.

\subsubsection{Válvula}

As válvulas são elementos que possuem a função de controlar a vazão dos fluidos, bem como assegurar a estanqueidade e segurança dos sistemas. As válvulas são modeladas de acordo com suas características de acionamento, em que, dependendo do tamanho e tipo teremos sua velocidade de abertura e de fechamento.

Segundo [1] podemos descrever matematicamente o modelo da válvula por:

$$
\frac{d h(t)}{d t}=-k \sqrt{h(t)+h_{0}}
$$

Onde h é o nível do reservatório e k é a constante da válvula. A constante da válvula $\mathrm{k}$ é determinada pela seguinte equação, como vemos em [1]:

$$
k_{n}=p_{n} \frac{S_{v \max } \sqrt{2 g}}{S_{T}} \quad \forall n=1,2,3 \ldots 7
$$

Onde $p_{i}$ é a posição da válvula e varia de $0 \leq p \leq 1, S_{v \max }$ é a área de abertura da válvula que em muitos casos devido a construção da válvula pode ser considerada a área da tubulação na qual a válvula está conectada, g é a aceleração da gravidade, $S_{T}$ é a área do reservatório e n representa o número de válvulas do sistema.

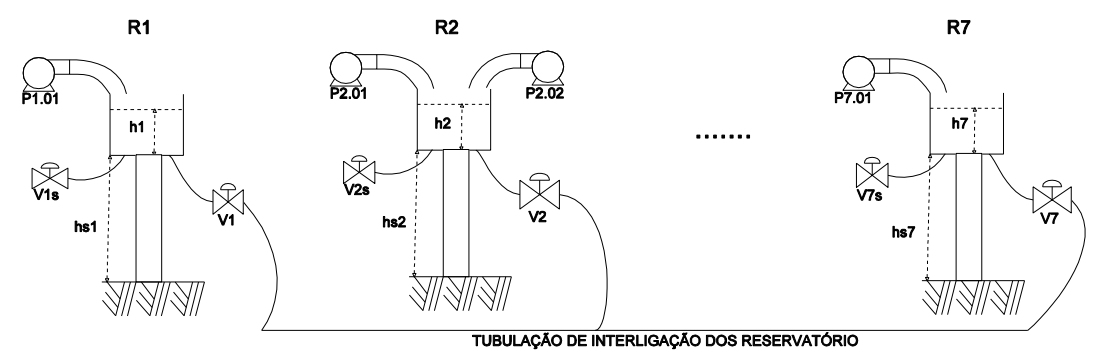

Figura 1: Esquema de ligação dos reservatórios.

\section{Modelo do Sistema}

Sabendo que os reservatórios são interligados e apresentam o mesmo desnível geométrico (linha piezométrica), podemos então obter suas equações com base no balanço 
4

de cargas de cada reservatório. Analisando suas vazões de entrada e saída, como vemos na figura 1 e usando as equações (4) e (6) podemos obter a seguinte equação diferencial para os níveis de cada reservatório:

$$
\begin{gathered}
\frac{d h_{n}(t)}{d t}=\frac{Q_{p n}(t)}{A_{b}}-K_{n} \sqrt{\left|2 . h_{n}-\sum_{1}^{7} h_{n}\right|} \cdot \operatorname{sign}\left(2 . h_{n}-\sum_{1}^{7} h_{n}\right)-K_{n s} \sqrt{h_{n}} \\
\forall n=\{1,2,3,4,5,6 \text { e } 7\}
\end{gathered}
$$

O modelo do sistema foi implementado computacionalmente no Matlab/Simulink [4].

\section{Controlador}

Para o controle do sistema é proposto o uso de um servosistema tipo 1, como visto em [5], modificado para aplicação em um sistema multivariável.

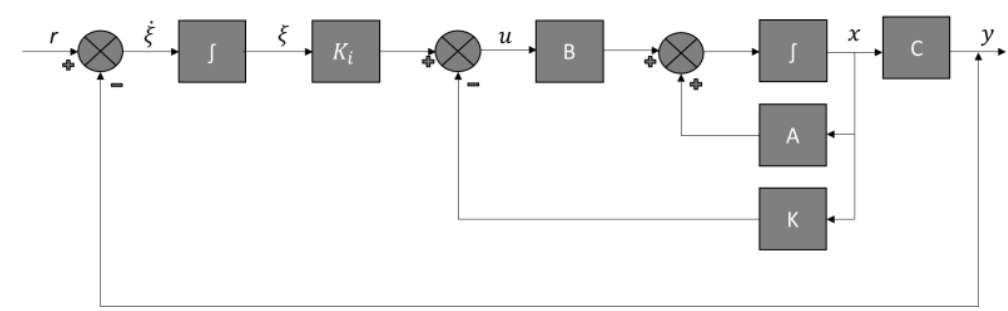

Figura 2: Diagrama de blocos do controlador.

Baseado no diagrama de figura 2, podemos descrever o controlador pelas seguintes equações:

$$
\begin{gathered}
\dot{x}=A x+B u \\
y=C x \\
u=-K x+K_{i} \xi \\
\dot{\xi}=r-y=r-C x
\end{gathered}
$$

Onde, $A, B$ e $C$ são os parâmetros do sistema, $x$ os estados do sistema, $y$ a saída do sistema, $r$ a referência de entrada e $u$ o sinal de controle aplicado ao sistema.

Combinando as equações (9) e (12) podemos descrever a dinâmica do sistema por:

$$
\left[\begin{array}{l}
\dot{x}(t) \\
\dot{\xi}(t)
\end{array}\right]=\left[\begin{array}{cc}
A & 0 \\
-C & 0
\end{array}\right]\left[\begin{array}{l}
x(t) \\
\xi(t)
\end{array}\right]+\left[\begin{array}{l}
B \\
0
\end{array}\right] u(t)+\left[\begin{array}{l}
0 \\
1
\end{array}\right] r(t)
$$

Se considerarmos o sistema assintoticamente estável, uma vez que $x(\infty)$, $\xi(\infty)$ e $u(\infty)$ tende a um valor constante, podemos definir as seguintes equações:

$$
\begin{array}{r}
x(t)-x(\infty)=x_{e}(t) \\
\xi(t)-\xi(\infty)=\xi_{e}(t) \\
u(t)-u(\infty)=u_{e}(t)
\end{array}
$$


Definindo um novo vetor para o erro $e(t)$,

$$
e(t)=\left[\begin{array}{l}
x_{e}(t) \\
\xi_{e}(t)
\end{array}\right]
$$

Podemos reescrever as equações em função do erro, segundo [5], como:

$$
\begin{gathered}
\dot{e}(\mathrm{t})=\widehat{A} e(t)+\widehat{B} u_{e}(t) \\
u_{e}(\mathrm{t})=-\widehat{K} e(\mathrm{t})
\end{gathered}
$$

Onde, $\widehat{A}=\left[\begin{array}{cc}A & 0 \\ -C & 0\end{array}\right], \widehat{B}=\left[\begin{array}{l}B \\ 0\end{array}\right]$ e $\widehat{K}=\left[\begin{array}{lll}K & \vdots & K_{i}\end{array}\right]$.

Substituindo (19) em (18),

$$
\dot{e}(\mathrm{t})=(\widehat{A}-\widehat{B} \widehat{K}) e(t)
$$

Para controlar o sistema não-linear usando um controlador linear, adotamos a estratégia de linearizar o modelo da planta em um ponto de operação, e obter os parâmetros $\widehat{K}$ usando dinâmica desejada por meio da alocação de polos assim podemos garantir, como vemos em (20), que o erro tenderá a zero nas condições próximas ao regime do sistema. Para o sistema apresentado as matrizes A, B e C são todas 7x7 e temos os sete estados (níveis dos reservatórios) sendo medidos.

\section{Resultados e Discussões}

Para a simulação utilizamos dados reais do sistema, como descritos na seção 2, para que a simulação se desse em condições bem próximas do sistema real.

Os resultados serão divididos em duas partes. Na primeira parte mostraremos o funcionamento do modelo da planta para as situações de abertura e fechamento das válvulas e a influência da bomba no sistema. Na segunda parte será mostrado o sistema com a ação do controlador.

\subsection{Simulação em malha aberta}

A resposta do modelo em malha aberta será mostrada apenas para três reservatórios, apesar da simulação conter os sete reservatórios, para que se seja possível visualizar bem a dinâmica das válvulas e a atuação das bombas. Na simulação a válvula de saída ficou $100 \%$ aberta, simulando a vazão total de abastecimento para o sistema, e foram impostas variações nas bombas e nas válvulas que interligam os reservatórios, como podemos ver nos gráficos dois e três da figura 3 .

Os resultados da simulação representam bem a dinâmica do sistema, pode-se ver na figura 3 que a abertura e fechamento das válvulas de interligação causa mudanças na dinâmica do sistema. Analisando o comportamento do nível $h_{3}$ percebe-se que após a abertura da válvula no instante 4 (horas) que o nível passa a subir, apesar da bomba manter sua vazão. Nesse instante ele passa a receber água dos outros reservatórios do sistema, já no instante 8 e 16 (horas), com a mudança da contribuição da bomba no sistema pode-se ver o nível se mantendo estável e logo em seguida subindo.

A bomba possui uma forte influência no sistema, uma explicação para isso, é que sua 
6

vazão é bem maior que a vazão das válvulas, por isso, para que se pudesse visualizar bem os gráficos de resposta da dinâmica, utilizamos um valor baixo para a vazão da bomba.
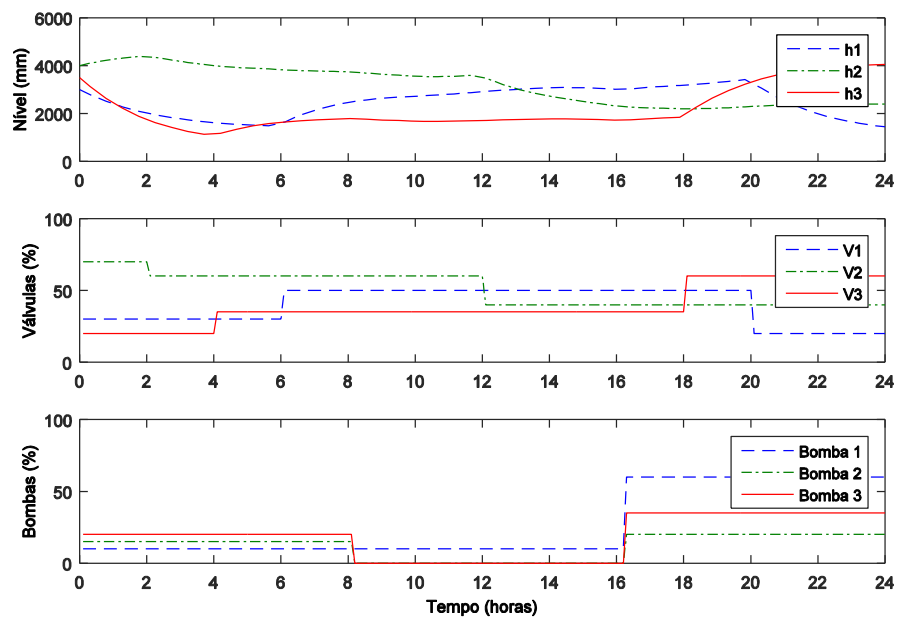

Figura 3: Simulação do modelo.

\subsection{Controlador}

O projeto do controlador foi baseado em um modelo linearizado em torno dos seguintes pontos de operação: Válvula de saída: $100 \%$, Vazão da bomba: $50 \%$, nível do reservatório 1: $2000 \mathrm{~mm}$, nível do reservatório 2: $5500 \mathrm{~mm}$, nível do reservatório 3: 3100 $\mathrm{mm}$, nível do reservatório 4: $3400 \mathrm{~mm}$, nível do reservatório 5: $5120 \mathrm{~mm}$, nível do reservatório 6: $4063 \mathrm{~mm}$, nível do reservatório 7: $3000 \mathrm{~mm}$. A variável manipulada no processo é a válvula de interconexão dos reservatórios e a controlada são os níveis.

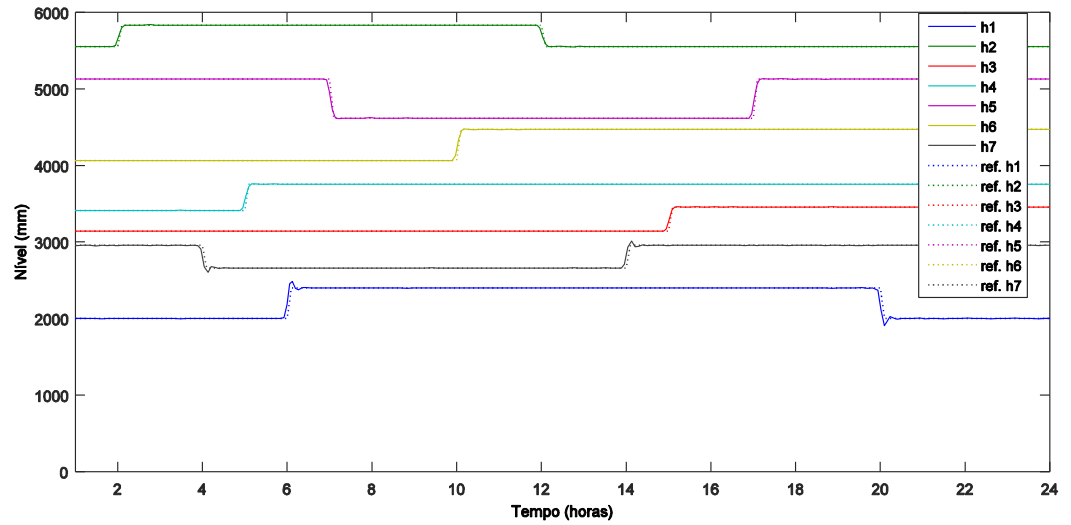

Figura 4: Modelo com servo controlador multivariável.

O modelo linearizado foi obtido a partir da função linmod do Matlab [4]. Os polos em malha fechada alocados foram: $(-0.1821,-1.1361,-1.1452,-1.1558,-1.1941,-1.1842$, -1.1654). Na simulação foram utilizadas referencias do tipo degrau (tracejado, figura 4).

Verifica-se na figura 4 que o controlador consegue impor o seguimento das referências sem erro, com uma convergência rápida para o valor de regime permanente. Pode-se perceber na variação das referências um pequeno acoplamento entre as variáveis, isso devido a interferência dos níveis em cada reservatório. Na figura 5 é apresentado o 
sinal de controle onde podemos ver a ação do controlador a cada mudança de referência.

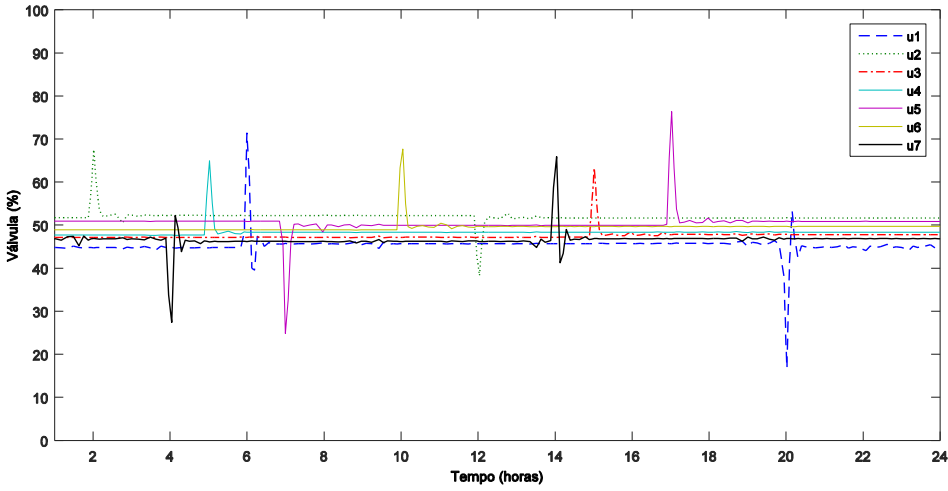

Figura 5: Sinal de controle aplicados nas válvulas.

\section{Conclusão}

Verificou-se por meio de simulação que de forma coerente o modelo apresentado descreve o comportamento de um sistema hidráulico, o que deverá em trabalho futuro ser ajustado e validado com os dados reais do sistema.

O controlador linear multivariável respondeu a dinâmica dentro de um ponto de operação para um sistema não-linear, o que aponta para a viabilidade na sua implementação pratica, mas em trabalhos futuros deverá investigada uma estratégia de controle não-linear que torne o desempenho do sistema menos dependente do ponto de operação.

\section{Agradecimentos}

Os autores agradecem ao Programa de Pós-Graduação em Engenharia Elétrica e de Computação (PPGEEC) e a UFRN pelo apoio e incentivo na realização das pesquisas.

\section{Referências}

[1] P. Chalupa and J. Novak. Modeling and model predictive control of a nonlinear hydraulic system. Computers and Mathematics with Applications 66, 155-164, (2013).

[2] I. Eker and T. Kara. Operation and Control of a Water Supply System, Isa Transaction, Vol. 42, 461-473, (2003)

[3] International Benchmarking Network For Water And Sanitation Utilities 2014 IBNET. www.ib-net.org. (2015).

[4] MATLAB. Version 7.10.0 (R2010a), The Math Works Inc., Natick, Massachusetts, EUA, (2010).

[5] K. Ogata, Engenharia de Controle Moderno, Editora Pearson Education, 5 Edição, (2010). 